\title{
Beyond mean-field description of the low-lying spectrum of ${ }^{16} \mathrm{O}$
}

\author{
M. Bender and P.-H. Heenen \\ Service de Physique Nucléaire Théorique et de Physique Mathématique \\ Université Libre de Bruxelles - C.P. 229, B-1050 Brussels, Belgium
}

\begin{abstract}
Starting from constrained Skyrme-mean-field calculations, the low-energy excitation spectrum of ${ }^{16} \mathrm{O}$ is calculated by configuration mixing of particle-number and angular-momentum projected mean-field states in the framework of the Generator Coordinate Method. Without any adjustable parameters, this approach gives a very good description of those states and their transition moments that can be described with our restriction to axially and reflection-symmetric shapes. The structure of low-lying $0^{+}$states is analyzed in terms of self-consistent $0 \mathrm{p}-0 \mathrm{~h}, 2 \mathrm{p}-2 \mathrm{~h}$, and $4 \mathrm{p}-4 \mathrm{~h}$ Hartree-Fock states.
\end{abstract}

Key words:

PACS: 21.10.Ky, 21.30.-n, 21.60.Jz, 27.30+t

\section{Introduction}

The doubly-magic oxygen isotope ${ }^{16} \mathrm{O}$ has always attracted much interest in nuclear structure physics. Its first excited $0^{+}$state has been interpreted by Morinaga [1] as an evidence for shape coexistence. Multi-nucleon transfer reactions have lead to the conclusion that the presence of a state corresponding to a deformed shape is due to excitations of both proton and neutron pairs across the $N$ and $Z=8$ closed shells. Several other excited states at low excitation energies can be interpreted as due to multi-particle-hole excitations (see the discussion in the Wood et al. review paper [2]). The first excited $0^{+}$ state is the head of a rotational band.

Shell model and mean-field calculations were already performed in the sixties $[3,4,5]$ with a description of this $0^{+}$state based on a deformed $4 \mathrm{p}-4 \mathrm{~h}$ configuration. The cluster model [6], using an empirical $\alpha$ - $\alpha$ interaction, qualitatively 
explains the deformed structure of the excited states. To go beyond a qualitative understanding of the ${ }^{16} \mathrm{O}$ spectrum appeared however very quickly as a difficult challenge. Mean-field calculations with more modern effective interactions $[7,8,9]$ clearly showed that correlations beyond the Hartree-Fock (HF) approach have to be included to describe successfully the first excited $0^{+}$state. Two kinds of correlations were mainly invoked: pairing correlations, and restoration of rotational symmetry which both leave the spherical configuration unchanged and lower the energy of the deformed configurations.

More recently, shell model calculations mixing $(0+2+4) \hbar \omega$ excitations $[10,11]$ have confirmed the schematic model of Brown and Green [4], and showed that the large quadrupole moment of the deformed configuration appears naturally in a full shell model diagonalization. It has also been possible in such calculations to test how well ${ }^{16} \mathrm{O}$ can be considered as an inert core as it is usually done in shell model calculations. The $0 \mathrm{p}-0 \mathrm{~h}$ component of the ground-state wave function was found to have a weight of the order of 40 to $50 \%$ only, with nearly equal importance of the $2 \mathrm{p}-2 \mathrm{~h}$ components.

The question of a correct description of the ${ }^{16} \mathrm{O}$ spectrum starting from a mean-field approach is thus still an open question. This nucleus is an anchor point where many approaches to the nuclear many-body problem can be compared. Indeed, besides the already mentioned mean-field and shell model calculations using effective interactions, there are also calculations based on so-called "realistic" nucleon-nucleon interactions (introduced as effective potentials to describe the phase shifts in nucleon-nucleon scattering), either already performed for ${ }^{16} \mathrm{O}[12]$, or to be expected for the near future [13].

In this paper, we present an application of the method introduced by Valor et al. [14], which performs a configuration mixing of projected self-consistent mean-field states, to calculate the low-energy spectrum of ${ }^{16} \mathrm{O}$, and to analyze its structure in terms of $n \mathrm{p}-n \mathrm{~h}$ Hartree-Fock states.

\section{The Method}

The starting point of the method is a set of BCS states $|q\rangle$ generated by constrained Skyrme-mean-field calculations. We have used the Skyrme parametrization SLy4 [15] together with a like-particle $T=1$ density-dependent zero-range pairing interaction [16]. The pairing strength adjusted in [17] has been reduced from $V=-1250 \mathrm{MeV} \mathrm{fm}^{3}$ to $V=-1000 \mathrm{MeV} \mathrm{fm}^{3}$ for both protons and neutrons, as in [14]. The pairing active space is limited by a soft cutoff at $5 \mathrm{MeV}$ above and below the Fermi energy [18]. This combination of effective interactions has been proven to be very successful in the description of a large number of experimental data all over the chart of nuclei. 
The set of mean-field wave functions $|q\rangle$ is generated by calculations with a constraint on the axial quadrupole moment $Q_{0}$. To avoid a collapse of pairing correlations along the constraining path, the approximate variation-afterprojection Lipkin-Nogami (LN) method is used. The Lipkin-Nogami prescription to evaluate an energy correction due to particle number projection is known to have deficiencies when pairing correlations are weak [19]. We do not make use of this correction, however, but introduce the LN method solely to ensure that pairing correlations, although weak, are present even in the spherical configuration. With that, the LN method provides BCS wave functions which are a fair starting point for an exact projection on particle number. Since these projected BCS wave functions are used further as basis functions for a configuration mixing, the deficiencies of the LN method should not affect the results.

The most important symmetries broken by the mean-field approach are restored after variation by standard projection techniques [14]. For each value of the quadrupole moment, the wave functions are projected simultaneously on angular momentum and on neutron and proton particle numbers, decomposing a given intrinsic BCS state $|q\rangle$ into wave functions $|J q\rangle$ corresponding to several values of the angular momentum. For each value of $J$ separately, these sets of non-orthogonal states $|J q\rangle$ are then mixed as a function of the quadrupole moment to give the final wave functions $|J k\rangle$, which correspond to collective states of the nucleus. A discrete number of values of the quadrupole moment are considered, but in such a way that the results do not depend on the discretization and are equivalent to a mixing on a continuous variable as in the generator coordinate method (GCM) [20,21]. Among the configurationmixed states $|J k\rangle$ so obtained, the physically interesting ones are the ground state $(J=0, k=0)$ and the few excited states which can be described by an axial quadrupole collective mode. States corresponding to modes that require a breaking of reflection symmetry (like $\alpha-{ }^{12} \mathrm{C}$ configurations or the tetrahedron-like 4- $\alpha$ configuration) are not included in our description of ${ }^{16} \mathrm{O}$.

\section{Projection}

The energy curves are shown in figure 1 . The mean-field curve, projected on particle numbers $N=Z=8$ only, shows, as expected, a deep minimum for the spherical configuration and no secondary minima. Two inflection points, however, are present at large deformations $\left(80\right.$ and $150 \mathrm{fm}^{2}$ ). Looking at the single-particle level scheme plotted for neutrons in figure 2, one sees that these points correspond to level crossings. At a $\beta_{2}$ value around 0.8 (or a mass quadrupole moment equal to $80 \mathrm{fm}^{2}$ ), a $d_{5 / 2}$ level crosses the $p_{1 / 2}$ level and is located below the Fermi level for larger quadrupole moments. Since due to time-reversal invariance, the levels are doubly degenerate and since 


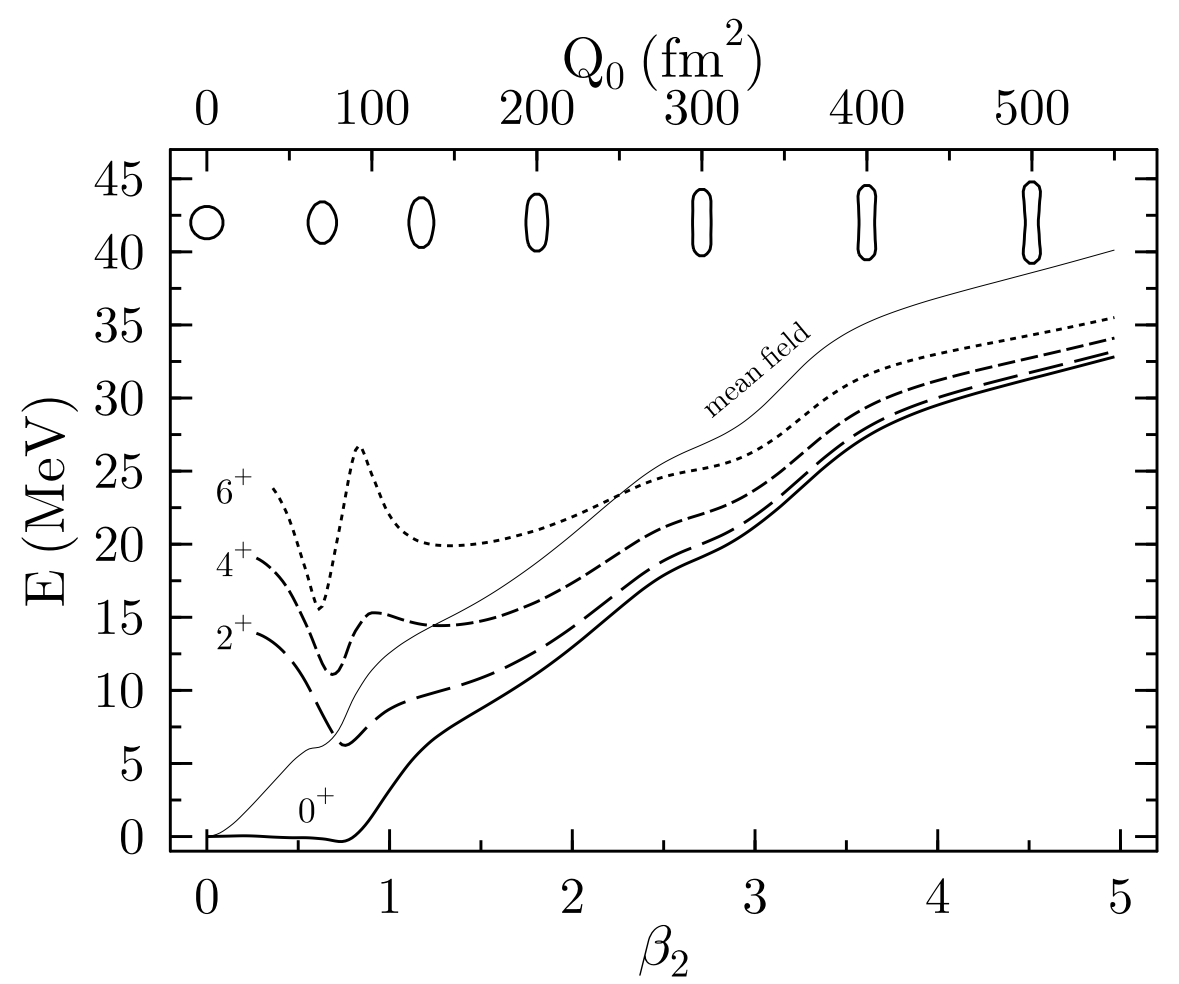

Fig. 1. Particle-number projected ("mean field") and particle-number and angular-momentum projected potential energy curves for ${ }^{16} \mathrm{O}$. All curves are drawn versus the (intrinsic) deformation of the BCS state which is projected. The upper axis gives the mass quadrupole moment $Q_{0}=\sqrt{16 \pi / 5}\left\langle r^{2} Y_{20}\right\rangle$, the lower the dimensionless deformation parameter $\beta_{2}=4 \pi\left\langle r^{2} Y_{20}\right\rangle /\left(3 R^{2} A\right)$ with $R=1.2 A^{1 / 3} \mathrm{fm}$. The various shapes along the paths are indicated by the contours of the total density at $\rho_{0}=0.07 \mathrm{fm}^{-3}$.

neutrons and protons have a very similar behavior in this $N=Z$ nucleus, the mean-field wave functions for $\beta_{2}$ values larger than 0.8 correspond to a deformed $4 \mathrm{p}-4 \mathrm{~h}$ configuration. Owing to pairing correlations, however, all the levels around the Fermi energy have sizable occupation probabilities. In the same way, for a $\beta_{2}$ value around 1.8, a crossing occurs between a state coming down from the $p f$ shell and a $p$ state. Note that for the protons the level crossings are at slightly smaller deformation than for the neutrons, a consequence of the Coulomb interaction. For extremely deformed shapes, above $\beta_{2}=4.0$, the mean-field configuration corresponds to a deformed $8 \mathrm{p}-8 \mathrm{~h}$ configuration which is associated to a chain of four $\alpha$ particles.

The energy curves projected on angular momentum, from $J=0$ to $J=6$, are also shown on figure 1 . While the spherical mean-field state has a $J=0$ component only, the deformed configurations can be projected on several angular momenta. Their $J=0$ components have always energies lower than the meanfield energy. Since for each quadrupole moment the mean-field energy is the weighted sum of the projected energies, figure 1 shows also that components with $J>6$ are dominant beyond $\beta_{2} \approx 2.5$. 


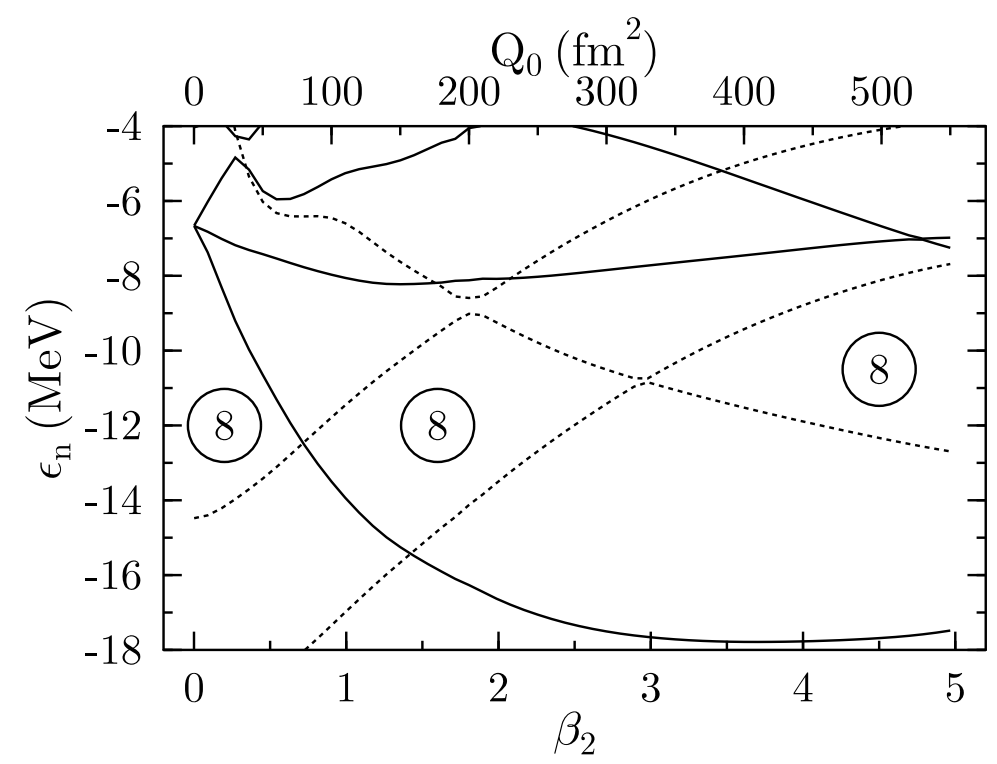

Fig. 2. Single-particle spectra for neutrons in ${ }^{16} \mathrm{O}$. Solid (dotted) lines represent levels with positive (negative) parity.

The $J=0$ curve is extremely flat up to the first single-particle level crossing. Beyond this point, the gain in energy due to angular momentum projection is approximately constant and of the order of $7 \mathrm{MeV}$. For angular momenta equal to 2 to 6 , a well-developed minimum is obtained for mean-field states with an axial quadrupole moment around $75 \mathrm{fm}^{2}$ which correspond to the deformation at which single-particle level crossing occurs. The shape of the mean-field density is also changing abruptly at this point, as can be seen on figure 1 and is reflected in the hexadecapole deformation. For a quadrupole moment value around $100 \mathrm{fm}^{2}$, the $\beta_{4}$ parameter, as defined in [22], changes sign. For still more deformed intrinsic wave functions, the projected energies vary very slowly, with soft minima obtained for $\beta_{2}$ values around 1.5.

\section{Configuration Mixing}

The configuration mixing of mean-field wave functions as performed in this work has several goals. The particle-number projection removes unwanted contributions coming from states with different particle numbers. The angular momentum projection separates the contribution from different angular momenta and generates wave-functions in the laboratory frame which provide transition probabilities and spectroscopic moments without further approximations. Finally, the variational configuration mixing with respect to a collective coordinate, the axial quadrupole moment in this work, removes the contributions to the ground state coming from collective vibrations and provides the excitation spectrum corresponding to this mode. 


\begin{tabular}{ccccccc}
\hline state & $\begin{array}{c}E_{\text {expt }} \\
(\mathrm{MeV})\end{array}$ & $\begin{array}{c}E_{\text {calc }} \\
(\mathrm{MeV})\end{array}$ & $\begin{array}{c}r_{\text {rms }} \\
(\mathrm{fm})\end{array}$ & $\begin{array}{c}Q_{s} \\
\left(\mathrm{e} \mathrm{fm}^{2}\right)\end{array}$ & $\begin{array}{c}Q_{0}(s) \\
\left.(\mathrm{e} \mathrm{fm})^{2}\right)\end{array}$ & $\beta_{2}(s)$ \\
\hline $0_{1}^{+}$ & 0.00 & 0.00 & 2.75 & & & \\
$0_{2}^{+}$ & 6.05 & 6.03 & 2.90 & & & \\
$2_{1}^{+}$ & 6.92 & 7.09 & 2.91 & -11.7 & 41.0 & 0.74 \\
$0_{3}^{+}$ & 12.05 & 12.45 & 3.32 & & & \\
$2_{2}^{+}$ & 13.02 & 13.18 & 3.22 & -22.9 & 80.2 & 1.4 \\
$0_{4}^{+}$ & - & 20.73 & 3.85 & & & \\
$2_{3}^{+}$ & - & 23.06 & 3.82 & -44.4 & 155 & 2.8 \\
\hline
\end{tabular}

Table 1

Experimental $\left(E_{\text {expt }}\right)$ and calculated $\left(E_{\text {calc }}\right)$ excitation energies for the low-lying $0^{+}$ and $2^{+}$states in ${ }^{16} \mathrm{O}$. Only states that can be described by our calculation are indicated. Also given are the calculated proton $\mathrm{rms}$ radii $r_{\mathrm{rms}}$, spectroscopic quadrupole moments $Q_{s}$ (in the laboratory frame), proton quadrupole moments $Q_{0}(s)$ and $\beta_{2}(s)$ deformation parameters in the intrinsic frame. Experimental excitation energies are taken from [23].

Results for the lowest states obtained in the configuration mixing calculation are given in table 1 and are compared with the experimental excitation energies. The calculated excitation spectrum is compared to the experimental data in figure 3 and to the mean-field energy curve in figure 4 . Only prolate configurations have been included. The projected oblate configurations have an overlap close to 1.0 with the spherical configuration which makes the configuration mixing calculation numerically unstable. The rms radii and de-

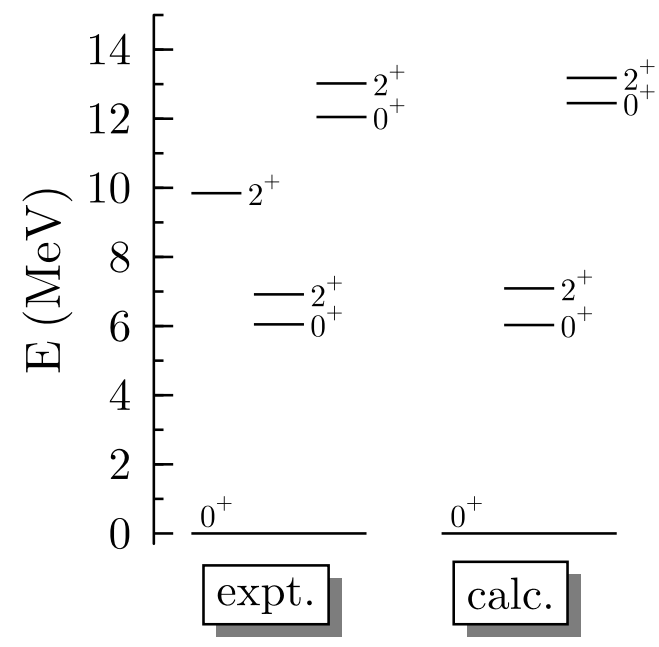

Fig. 3. Comparison between the experimental and theoretical low-lying $0^{+}$and $2^{+}$ states in ${ }^{16} \mathrm{O}$. 


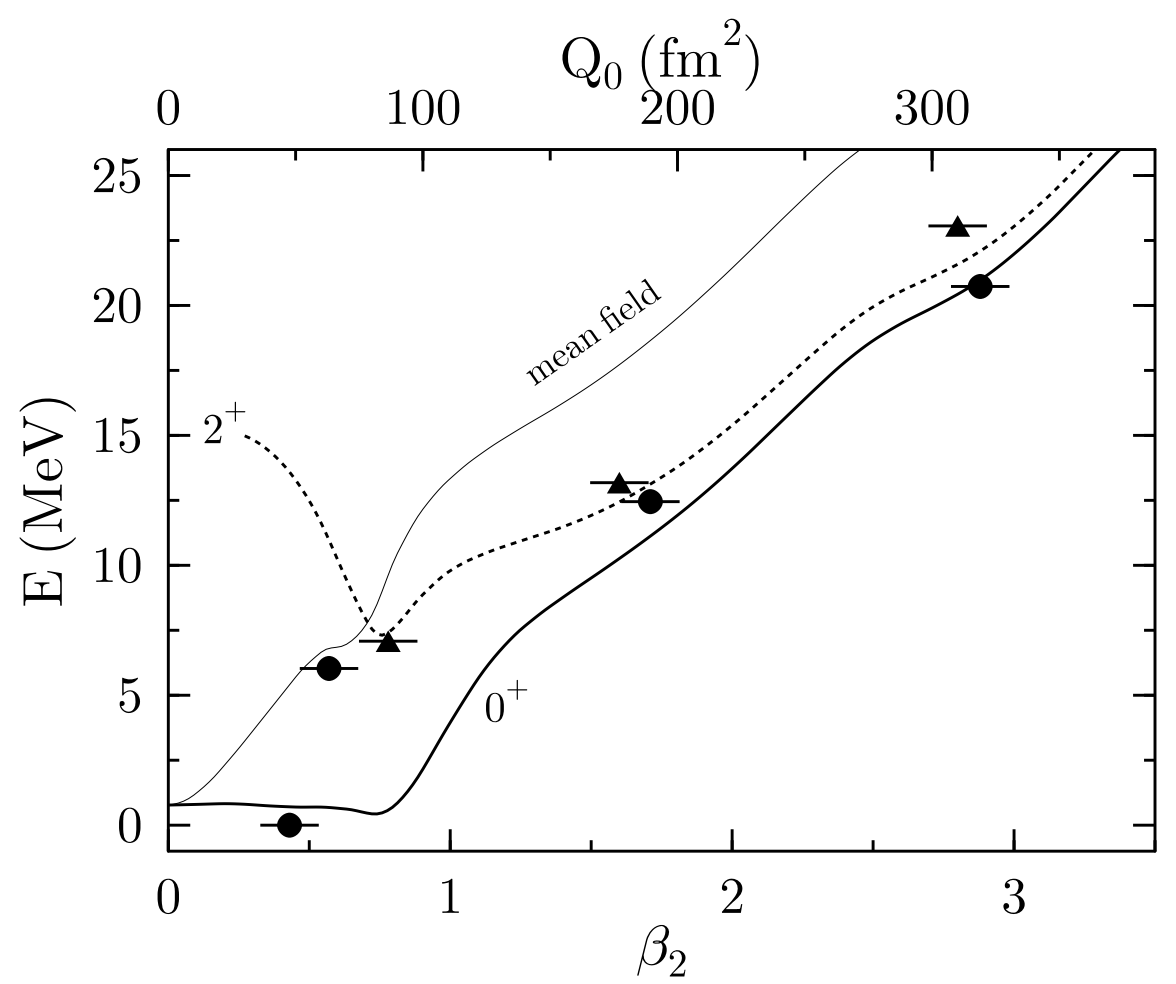

Fig. 4. Spectrum of the lowest $0^{+}$(circles) and $2^{+}$(triangles) states, plotted at a deformation corresponding to the average deformation of the intrinsic wave functions. These averages are determined using the weight of each mean-field state in the collective wave functions. Also shown are the angular momentum projected energy curves and the (particle-number projected only) "mean-field" curve (thin black line).

formations given in table 1 are calculated from the point-proton distribution and do not include the corrections that have to be introduced to calculate charge radii that can be compared with the experimental data, see e.g. [24].

With our method, one obtains automatically the spectroscopic quadrupole moments $Q_{s}$ in the laboratory frame. The charge quadrupole moment in the intrinsic frame $Q_{0}(s)$ is then calculated with the relation $Q_{0}(s)=-Q_{s}(2 J+3) / J$ assuming $K=0$ states [25]. The dimensionless deformation parameter $\beta_{2}$ is related to $Q_{0}(s)$ by $\beta_{2}(s)=\sqrt{5 /(16 \pi)} \times 4 \pi Q_{0}(s) /\left(3 R^{2} Z e^{2}\right)$.

The assignment of the calculated $0_{2}^{+}$and $2_{1}^{+}$states to experiment in table 1 is tentative. Note that the restriction of our calculation to reflection-symmetric configurations does not permit to describe states with negative parity or whose intrinsic structure is supposed to be asymmetric. For instance, the $\alpha_{-}{ }^{12} \mathrm{C}$ configuration assigned to the experimental $2^{+}$state at $9.8440 \mathrm{MeV}$ [23] cannot be represented by our model. For the other states, the agreement between the experimental and theoretical energies is excellent.

The correlations introduced by the configuration mixing decrease the en- 


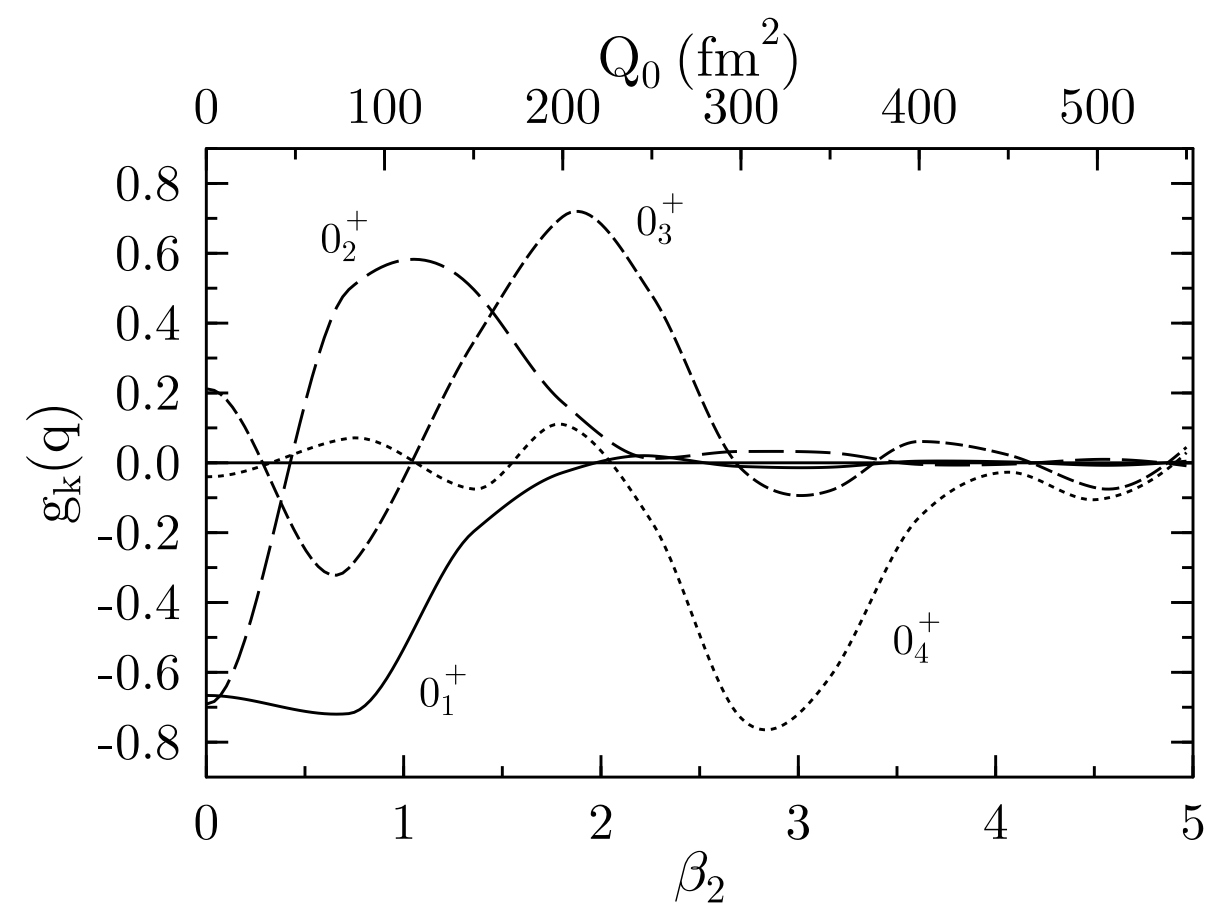

Fig. 5. GCM wave functions $|J k\rangle=\sum_{q} g_{k}(q)|J q\rangle$ of the four lowest $0^{+}$states.

ergy of the $0_{1}^{+}$state with respect to the $\mathrm{HF}$ ground state by $2.3 \mathrm{MeV}$. This gain in energy, although significant, is lower than in several of our previous configuration-mixing calculations. In ${ }^{208} \mathrm{~Pb}$, for instance, removing quadrupole vibrations from the mean-field ground state by configuration mixing without angular momentum projection brings already 2.0 MeV of extra energy [26]. In ${ }^{24} \mathrm{Mg}$, restoration of symmetries and the inclusion of the contribution from quadrupole vibrations decrease the mean-field ground-state energy by more than $5 \mathrm{MeV}[14]$.

The mixing coefficients $g_{k}(q)$ for the four lowest $0^{+}$states are plotted in figure 5 . The ground-state wave function is spread over a large range of deformations, with nearly equal weight up to a $\beta_{2} \approx 0.8$. Its rms radius $r_{\mathrm{rms}}=2.75 \mathrm{fm}$ is therefore significantly larger than the $2.68 \mathrm{fm}$ value of the spherical HF state. The maximum weight for the first excited state is obtained around $\beta_{2}$ equal to 1.0, but with significant components at lower quadrupole moments. The third and fourth $0^{+}$states are shifted to configurations with larger deformations. As can be seen from the mean deformation of their mean-field components (see Fig. 4), and the shapes displayed in Fig. 1, none of them corresponds to a chain of $4 \alpha$ particles which occurs at even larger deformation around $\beta_{2} \approx 4.0$.

The $B(E 2)$ values for transitions from the $2_{1}^{+}$to the ground state and to the first excited $0^{+}$state are well described by our model. The error is larger for the transition from the $2_{2}^{+}$state to the ground state. This is not surprising since important configurations like $\alpha-{ }^{12} \mathrm{C}$ which are not included in our model 


\begin{tabular}{|c|c|c|c|c|c|c|c|}
\hline \multirow[t]{2}{*}{ transition } & \multicolumn{2}{|c|}{$B(E 2) \uparrow /\left(e^{2} \mathrm{fm}^{4}\right)$} & \multirow{2}{*}{$\begin{array}{c}Q_{0}(t) \\
\left(e \mathrm{fm}^{2}\right)\end{array}$} & \multirow[t]{2}{*}{$\beta_{2}(t)$} & \multirow[t]{2}{*}{ transition } & \multicolumn{2}{|c|}{$M(E 0) / \mathrm{fm}^{2}$} \\
\hline & expt. & calc. & & & & expt. & calc. \\
\hline $2_{1}^{+} \rightarrow 0_{1}^{+}$ & $42 \pm 1.4$ & 38 & 19.7 & 0.36 & $0_{2}^{+} \rightarrow 0_{1}^{+}$ & $3.55 \pm 0.21$ & 5.735 \\
\hline $2_{2}^{+} \rightarrow 0_{1}^{+}$ & $21 \pm 7$ & 4.7 & 6.9 & 0.12 & $0_{3}^{+} \rightarrow 0_{1}^{+}$ & $4.03 \pm 0.09$ & 0.690 \\
\hline $2_{1}^{+} \rightarrow 0_{2}^{+}$ & $370 \pm 4$ & 241 & 49.2 & 0.89 & & & \\
\hline $2_{2}^{+} \rightarrow 0_{2}^{+}$ & $42 \pm 7$ & 74 & 27.3 & 0.49 & & & \\
\hline $2_{2}^{+} \rightarrow 0_{3}^{+}$ & - & 590 & 77.0 & 1.39 & & & \\
\hline
\end{tabular}

Table 2

Reduced transition probabilities $B(E 2) \uparrow$, calculated transition quadrupole moments $Q_{0}(t)=\sqrt{16 \pi B(E 2) \uparrow / 5}$, corresponding deformation parameters $\beta_{2}(t)$, and monopole matrix transition elements $M(E 0)=\left\langle f\left|\sum_{p} r_{p}^{2}\right| i\right\rangle$ between low-lying states in ${ }^{16} \mathrm{O}$. The experimental data are taken from [23].

should play a role at the excitation energy of the $2_{2}^{+}$state. For a similar reason, the monopole transition probabilities are in better agreement with experiment for the first excited $0^{+}$state than for the second.

Table 2 lists the experimental value from [23]. The values adopted by Raman et al. [27] for the $B(E 2) \uparrow=(40.6 \pm 3.8) e^{2} \mathrm{fm}^{4}$ of the $2_{1}^{+} \rightarrow 0_{1}^{+}$transition is even in better agreement with our result. The $Q_{0}(t)$ of the transitions within a rotational band, i.e. $2_{1}^{+} \rightarrow 0_{2}^{+}$and $2_{2}^{+} \rightarrow 0_{3}^{+}$, are close to the intrinsic quadrupole moments $Q_{0}(s)$ of the $2^{+}$states involved as given in table 1 .

\section{Analysis of the Collective States}

Analyses of the shell model wave functions obtained in a $(0+2+4) \hbar \omega$ active space $[10,11]$ have shown that the ${ }^{16} \mathrm{O}$ ground-state wave function is by far not a pure $0 \hbar \omega$ state and that the breaking of the closed shells at $N$ and $Z$ equal to 8 is large. The $0 \hbar \omega$ component of the shell-model wave function has a weight of the order of $50 \%$ only, with components of comparable importance in the $2 \hbar \omega$ subspace. The first excited $0^{+}$state has an energy very close to the first experimental $0^{+}$and is composed to $90 \%$ of a $4 \mathrm{p}-4 \mathrm{~h}$ configuration.

It is not evident to perform a similar analysis for self-consistent wave functions. In our model, there is no spherical oscillator basis on which the individual wave functions are expanded. However, it is important to find a common language with shell-model calculations and to interpret a deformed mean-field basis in terms of a spherical basis. In the framework of self-consistent mean-field models, a natural choice for the reference state is provided by the self-consistent 
Hartree-Fock (HF) 0p-0h ground state, which is of course spherical for the closed-shell nucleus ${ }^{16} \mathrm{O}$. To build $n \mathrm{p}$ - $n \mathrm{~h}$ excitations in an HF framework requires some care. They could be constructed non-self-consistently on top of the spherical HF state by just changing the occupation of the single-particle levels below and above the Fermi surface. However, such excitations are not unique and the resulting wave function is, in general, not spherical. A much more natural procedure is to consider the deformation also in the $n \mathrm{p}-n \mathrm{~h}$ HF states and to construct them self-consistently by solving the HF equations with appropriate occupation numbers of the single-particle states. These $4 \mathrm{p}-4 \mathrm{~h}$ and $8 \mathrm{p}-8 \mathrm{~h}$ states have deformations corresponding to the deformed shell closures that can be seen around $\beta_{2} \approx 1.4$ and $\beta_{2} \approx 4.5$ in figure 2 . Each fully self-consistent $n$ p$n \mathrm{~h}$ HF state $|n \mathrm{p}-n \mathrm{~h}\rangle$ defines a basis of deformed single-particle states different from the other excitations. As a consequence, the various $|n \mathrm{p}-n \mathrm{~h}\rangle$ are not orthonormal. However, since of their very different structure (deformation and $n \mathrm{p}-n \mathrm{~h}$ excitations), their overlap is extremely small and can be neglected.

Since they are deformed, the $n \mathrm{p}-n \mathrm{~h}$ HF states are not eigenstates of angular momentum and projected $n \mathrm{p}-n \mathrm{~h}$ states $|J n \mathrm{p}-n \mathrm{~h}\rangle$ are constructed by symmetry restoration.

We analyze the $n \mathrm{p}-n \mathrm{~h}$ content of the collective states $|J k\rangle$ by calculating the amplitude $|\langle J k \mid J n \mathrm{p}-n \mathrm{~h}\rangle|^{2}$. Note that our procedure is unambiguous and can be used to analyze any state constructed from mean-field wave functions. It permits to quantify the differences between simple mean-field configurations and the fully projected configuration mixing wave functions. The way $n \mathrm{p}-$ $n \mathrm{~h}$ excitations are constructed is not the same as in shell model calculations and the single-particle wave functions do not have the same analytical form (the self-consistent wave functions discretized on a mesh have a much better asymptotic behavior than oscillator wave functions). Nevertheless, our procedure will give us some insights on the relation between our wave functions and shell model ones.

The properties of the HF and projected HF states are given in table 3 where we also indicated the weight of the projected HF states in each of the four lowest collective $0^{+}$states.

From figure 6, one clearly sees that there is a correlation between the deformation of the projected mean-field state and its overlap with the $n \mathrm{p}-n \mathrm{~h}$ configurations. One sees also from table 3 that the deformation of the $n \mathrm{p}-n \mathrm{~h}$ HF states increases rapidly with the number $n$ of excitations.

With about $75 \%$ weight of the $0 \mathrm{p}-0 \mathrm{~h}$ HF state, see table 3 , the breaking of the closed shell character of the $0_{1}^{+}$ground-state wave function is not as large as in shell model calculations, but still sizable. Note that a part of the admixture of higher shells obtained in shell-model calculations is due to the fact that 


\begin{tabular}{lccccccccc}
\hline state & $E_{\mathrm{HF}}$ & $E_{J \mathrm{HF}}$ & $Q_{0}$ & $\beta_{2}$ & \multicolumn{4}{c}{$|\langle J k \mid J n \mathrm{p}-n \mathrm{~h}\rangle|^{2}$} \\
\cline { 7 - 9 } & & $(\mathrm{MeV})$ & $(\mathrm{MeV})$ & $\left(\mathrm{fm}^{2}\right)$ & & $0_{1}^{+}$ & $0_{2}^{+}$ & $0_{3}^{+}$ & $0_{4}^{+}$ \\
\hline 0p-0h & 0.0 & 0.0 & 0 & 0.00 & 0.737 & 0.195 & 0.006 & 0.0001 \\
2p-2h (p) & 14.92 & 11.96 & 41 & 0.37 & 0.094 & 0.098 & 0.018 & 0.003 \\
2p-2h (n) & 15.20 & 12.09 & 41 & 0.37 & 0.099 & 0.098 & 0.019 & 0.002 \\
4p-4h & 15.95 & 11.26 & 119 & 1.08 & 0.027 & 0.212 & 0.193 & 0.003 \\
8p-8h & 38.03 & 31.48 & 466 & 4.21 & 0.000 & 0.000 & 0.000 & 0.034 \\
\hline
\end{tabular}

Table 3

Excitation energy of the $\mathrm{HF}\left(E_{\mathrm{HF}}\right)$ and $J=0$ projected HF states $\left(E_{J \mathrm{HF}}\right)$ with respect to the $(0 \mathrm{p}-0 \mathrm{~h}) \mathrm{HF}$ ground state. The mass quadrupole moment $Q_{0}$ and the deformation $\beta_{2}$ of the self-consistent $0 \mathrm{p}-0 \mathrm{~h}, 2 \mathrm{p}-2 \mathrm{~h}, 4 \mathrm{p}-4 \mathrm{~h}$, and $8 \mathrm{p}-8 \mathrm{~h}$ HF states are also given. The last four columns give the weights $|\langle J k \mid J n \mathrm{p}-n \mathrm{~h}\rangle|^{2}$ of their $J=0$ component in the four lowest $0^{+}$states $|J k\rangle$ obtained from the configuration mixing.

the "real" single-particle potential is not an harmonic oscillator as assumed in a shell model basis. Therefore it is not surprising that we find a smaller admixture of higher shells as we start with single-particle wave functions with a more realistic asymptotic behavior.

For the $4 \mathrm{p}-4 \mathrm{~h}$ configuration, where a pair of protons and neutrons is excited from the $1 p_{1 / 2}$ to the $2 d_{5 / 2}$ levels, a minimum in the HF energy is obtained for a mass quadrupole moment of $Q_{0}=119 \mathrm{fm}^{2}$, or $\beta_{2}=1.08$. This $4 \mathrm{p}-4 \mathrm{~h}$ configuration corresponds to the deformed $N=Z=8$ shell closure visible at about the same deformation in figure 2. The excitation energy of this deformed HF state (with respect to the spherical 0p-0h HF state), is equal to $15.95 \mathrm{MeV}$, far above the experimental value of $6.03 \mathrm{MeV}$ for the first excited $0^{+}$state. The restoration of rotational symmetry by projection on angular momentum $J=0$ decreases the calculated excitation energy to $11.25 \mathrm{MeV}$, still too high compared to experiment. To complete the analysis, we have also constructed $2 \mathrm{p}-2 \mathrm{~h}$ and $8 \mathrm{p}-8 \mathrm{~h}$ configurations. The only two $2 \mathrm{p}-2 \mathrm{~h}$ states that we have considered are obtained by promoting either a pair of protons or a pair of neutrons from the $p_{1 / 2}$ shell to the $d_{5 / 2}$ shell. As we include the Coulomb interaction self-consistently, these two states have slightly different energies. The $8 \mathrm{p}-8 \mathrm{~h}$ configuration corresponds to the occupation of the $s d$ and $p f$ states which are below the Fermi level at very large deformations (see figure 2).

The 0p-0h HF state is the dominant component of the collective ground state. If one adds the weights of the $0 \mathrm{p}-0 \mathrm{~h}, 2 \mathrm{p}-2 \mathrm{~h}$ and $4 \mathrm{p}-4 \mathrm{~h}$ states, one obtains a nearly complete description of the ground state, the missing components being probably $2 \mathrm{p}-2 \mathrm{~h}$ states not included in our analysis. One should, however, take into account that the self-consistent $n \mathrm{p}-n \mathrm{~h}$ states are not orthogonal and have 


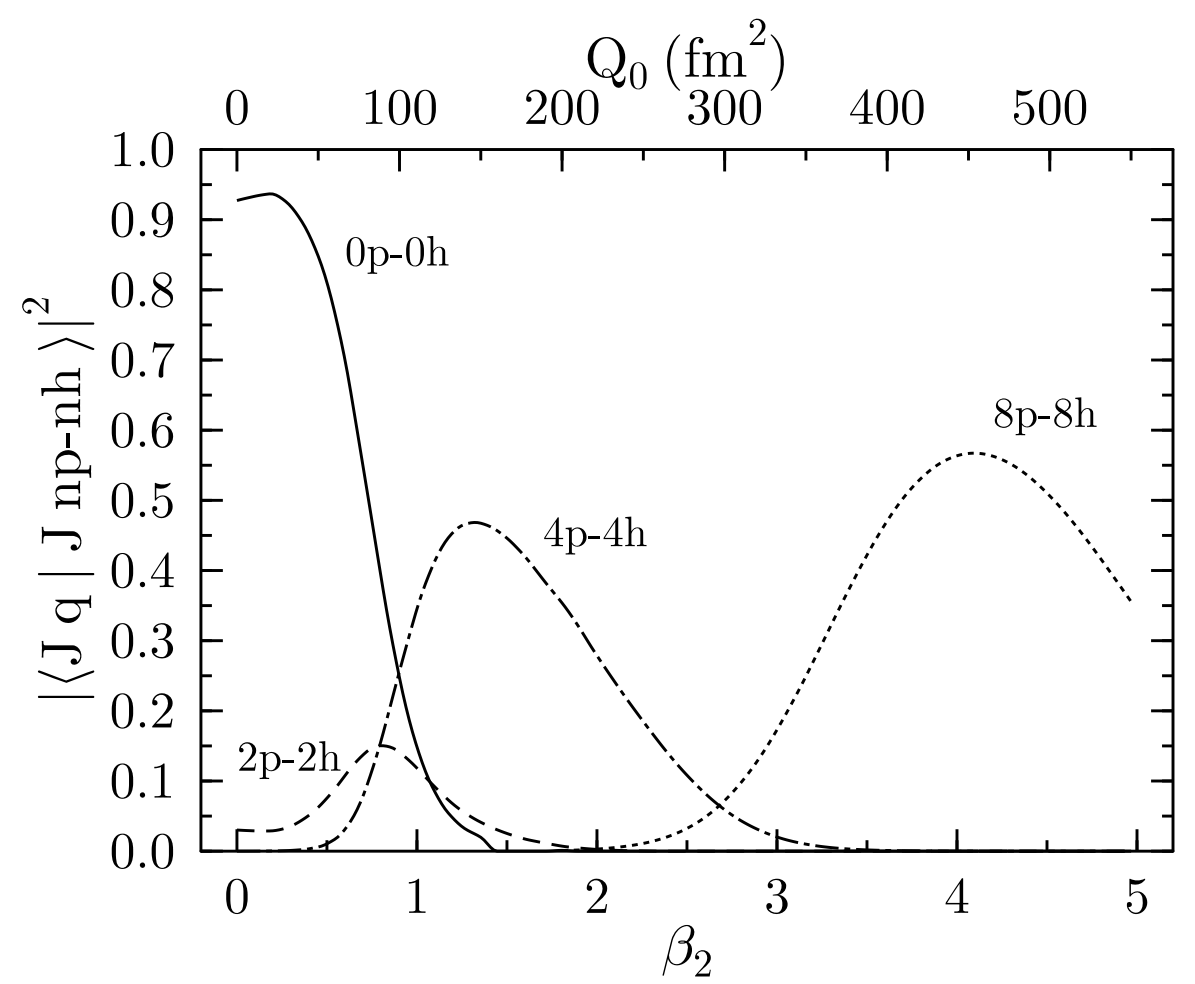

Fig. 6. Weight $|\langle J q \mid J n \mathrm{p}-n \mathrm{~h}\rangle|^{2}$ of the $J=0$ projected spherical $0 \mathrm{p}-0 \mathrm{~h}$ and deformed $2 \mathrm{p}-2 \mathrm{~h}, 4 \mathrm{p}-4 \mathrm{~h}$, and $8 \mathrm{p}-8 \mathrm{~h}$ HF states $|J n \mathrm{p}-n \mathrm{~h}\rangle$ in the $J=0$ projected paired mean-field states $|J q\rangle$. The weight of the two different $2 \mathrm{p}-2 \mathrm{~h}$ states is very similar, therefore just one is drawn.

small, but non-vanishing overlaps. With less than 3\%, the contribution from the pure HF $4 \mathrm{p}-4 \mathrm{~h}$ configuration to the ground state is small and lower than the shell-model values (between 4 and $12 \%$ depending on the interactions).

The excited $0_{2}^{+}$state has a much more complicate structure, without any dominant configuration. As can be seen in table 3 , the weights of the $0 \mathrm{p}-0 \mathrm{~h}, 2 \mathrm{p}-$ $2 \mathrm{~h}$ and $4 \mathrm{p}-4 \mathrm{~h}$ configurations are quite close. These four components, however, represent only about $60 \%$ of the full state. Looking to figure 5 , one can see that the spreading of this state extends up to deformations of the order of 200 $\mathrm{fm}^{2}$, where the overlap between the $n \mathrm{p}-n \mathrm{~h}$ HF states and the projected BCS states are small (see figure 6 ). Many other $n \mathrm{p}-n \mathrm{~h}$ configurations are therefore necessary to obtain a full description of this $0_{2}^{+}$state.

None of the collective states is dominated by the deformed $N=Z=8$ shell closure. The excited $0_{3}^{+}$state has also a small $4 \mathrm{p}-4 \mathrm{~h}$ component and is constructed on mean-field states with larger deformation than the 4p-4h HF state.

As can be seen from table 3 , the $8 \mathrm{p}-8 \mathrm{~h}$ state plays no role for the low-lying $0^{+}$states. The first state in which it has a large weight of 0.48 is the $0_{6}^{+}$ state. The density distribution of the $8 \mathrm{p}-8 \mathrm{~h}$ state is close to that of a four- $\alpha-$ chain configuration. Its $38.0 \mathrm{MeV}$ excitation energy is lowered to $32.2 \mathrm{MeV}$ 
by projection on angular momentum and to $32.0 \mathrm{MeV}$ by the configuration mixing. It is thus very large. This is, however, not inconsistent with the fact that there is up to now no convincing evidence for the presence of such a state in the low-energy spectrum of ${ }^{16} \mathrm{O}$.

\section{Summary and Conclusions}

This study of the doubly-magic nucleus ${ }^{16} \mathrm{O}$ has demonstrated the descriptive and predictive power of methods based on a self-consistent mean-field approach including correlations. In particular, the gain in energy of the pure 4p-4h HF state, that is brought first by projection on angular momentum, and then by mixing on the axial quadrupole moment, is impressive. This procedure does not only permit to obtain the first excited $0^{+}$state at the right energy, but also other excitations and transition matrix elements which are in reasonable agreement with available data. Somewhat surprisingly, the $4 \mathrm{p}-4 \mathrm{~h}$ $\mathrm{HF}$ state is evenly spread over the first and second excited $0^{+}$states, leaving none of them associated with a simple $4 \mathrm{p}-4 \mathrm{~h}$ configuration.

Our results are not very sensitive to the parametrizations of the mean-field and pairing interactions that are used. The Skyrme interaction SLy4 used here was adjusted at the mean-field level to binding energies, charge radii, and nuclear

matter properties of a few nuclei, among them ${ }^{16} \mathrm{O}$. The correlations taken into account in our study increase the binding energy by $2.3 \mathrm{MeV}$ compared to the spherical HF state, which leads now to a slight over-estimation of the ${ }^{16} \mathrm{O}$ ground state-energy. Similarly, the proton rms radius is slightly increased. Although it is gratifying to see that the change in total energy is below $2 \%$ while the change in radii stays below $3 \%$, it is clear that a readjustment of the effective interaction will have to be done when a larger experience will have been obtained on the effect of correlations.

We have included the most important symmetry restorations to permit to compare our results directly with experimental data in the laboratory frame of reference. Our calculations demonstrate that this is a key to a successful quantitative description of the low-energy states when starting from a meanfield approach. Some ingredients, however, are still missing in our model. There are still symmetries broken by our approach. Our wave functions break translational and Galilean invariances. The implicit assumption is made that these broken symmetries bring similar errors on all collective wave functions. To check this hypothesis in our model is unfortunately still beyond numerical possibilities. Projection on isospin seems to be the next natural step to enlarge the predictive power of the method even further. We have also not considered proton-neutron pairing correlations, although ${ }^{16} \mathrm{O}$ is an $N=Z$ nucleus. Developments in the understanding of these correlations and their modeling are still 
necessary. Concerning shape degrees of freedom, reflection-asymmetric configurations like ${ }^{12} \mathrm{C}+{ }^{4} \mathrm{He}$ still have to be incorporated to describe several states of astrophysical interest. We have also restricted ourselves so far to axially symmetric shapes. Including triaxiality might alter the results for high-lying excitations which spread differently into the $\beta-\gamma$ plane.

\section{Acknowledgments}

This research was supported in part by the PAI-P3-043 of the Belgian Office for Scientific Policy. We thank G. Bertsch and H. Flocard for fruitful and inspiring discussions. M. B. acknowledges support through a European Community Marie Curie Fellowship. P.-H. H. thanks the Institute for Nuclear Theory at the University of Washington for its hospitality during the completion of part of this work.

\section{References}

[1] H. Morinaga, Phys. Rev. 101 (1956) 254.

[2] J. L. Wood, K. Heyde, W. Nazarewicz, M. Huyse and P. Van Duppen, Phys. Rep. 215 (1992) 101.

[3] W. H. Bassichis and G. Ripka, Phys. Lett. 15 (1965) 320.

[4] G. E. Brown and A. M. Green, Nucl. Phys 75 (1966) 401.

[5] S. J. Krieger, Phys. Rev. Lett. 22 (1969) 97.

[6] G. Bertsch and W. Bertozzi, Nucl. Phys. A165 (1971) 199.

[7] M. Girod and B. Grammaticos, Phys. Rev. C 27 (1983) 2317.

[8] D. Auverlot, P. Bonche, H. Flocard and P.-H. Heenen, Phys. Lett. 149B (1984) 6.

[9] D. C. Zheng, D. Berdichevsky and L. Zamick, Phys. Rev. C 38 (1988) 437.

[10] W. C. Haxton and C. Johnson, Phys. Rev. Lett. 65 (1990) 1325.

[11] E. K. Waburton, B. A. Brown and D. J. Mollener, Phys. Lett. B293 (1992) 7.

[12] A. Fabracini, F. Arias de Saavedra and G. Co', Phys. Rev. C 61 (2000) 044302.

[13] P. Navratil, J. P. Vary and B. R. Barrett, Phys. Rev. Lett. 84 (2000) 5728.

[14] A. Valor, P.-H. Heenen and P. Bonche, Nucl. Phys. A671 (2000) 145. 
[15] E. Chabanat, P. Bonche, P. Haensel, J. Meyer and R. Schaeffer, Nucl. Phys. A635 (1998) 231, Nucl. Phys. A643 (1998) 441(E).

[16] J. Terasaki, P.-H. Heenen, H. Flocard and P. Bonche, Nucl. Phys. A600 (1996) 371.

[17] C. Rigollet, P. Bonche, H. Flocard and P.-H. Heenen, Phys. Rev. C 59 (1999) 3120 .

[18] P. Bonche, H. Flocard, P.-H. Heenen, S. Krieger and M. S. Weiss, Nucl. Phys. A443 (1985) 39.

[19] P. Magierski, S. Ćwiok, J. Dobaczewski and W. Nazarewicz, Phys. Rev. C 48 (1993) 1686.

[20] D. L. Hill and J. A. Wheeler, Phys. Rev. 89 (1953) 1102.

[21] P. Bonche, J. Dobaczewski, H. Flocard, P.-H. Heenen and J. Meyer, Nucl. Phys. A510 (1990) 466.

[22] S. Ćwiok, J. Dobaczewski, P.-H. Heenen, P. Magierski, W. Nazarewicz, Nucl. Phys. A611 (1996) 211.

[23] D. R. Tilley, H. R. Weller and C. M. Cheves, Nucl. Phys. A564 (1993) 1.

[24] M. Bender, P.-H. Heenen and P.-G. Reinhard, Rev. Mod. Phys., in print (2003).

[25] P. Ring and P. Schuck, The Nuclear Many Body Problem, Springer Verlag, Berlin, 1980.

[26] P.-H. Heenen, A. Valor, M. Bender, P. Bonche and H. Flocard, Eur. Phys. J. A11 (2001) 393.

[27] S. Raman, C. W. Nestor, Jr. and P. Tikkanen, Atom. Data Nucl. Data Tables 78 (2001) 1. 